\title{
Developmental Factors Contributing to the Susceptibility to Bradycardia in Isolated, Cultured Fetal Mouse Hearts
}

\author{
MICHAEL MAURER, JR. \\ Department of Medicine (Cardiology) and The Edward Mallinckrodt Department of Pediatrics Washington University \\ School of Medicine and the Division of Neonatology, St. Louis Children's Hospital, St. Louis, Missouri USA.
}

\begin{abstract}
Summary
A propensity for bradycardia in preterm human infants suggested that the heart rate response to cholinergic stimulation may vary during development. Isolated, intact fetal mouse hearts (FMH) in organ culture were used as a model to explore developmental differences in chronotropic response to cholinergic stimulation. FMH's of gestational ages (GA) from 13-22 days, maintained for $36 \mathrm{hr}$ in culture, were exposed to acetylcholine (AcH) with and without prior addition of physostigmine. Heart rate decreased markedly with $10^{-4}$ and $10^{-6} \mathrm{M} \mathrm{AcH} \mathrm{(84 \pm 3}$ and 48 $\pm 4 \%$ ) in 13-14 day hearts, but the decrease was progressively blunted with increasing age and was only $7 \pm 3$ and $3 \pm 2 \%$ at $21-$ 22 days GA. Physostigmine markedly enhanced the cholinergic response in older hearts with $54 \pm 4$ and $32 \pm 5 \%$ decreases in heart rate with the two doses of AcH at 21-22 days. However, it did not alter the response in younger hearts.

The data suggest that the chronotropic response to AcH progressively diminishes with advancing GA and the extent of intrinsic cholinesterase activity at different GA's is, in part, responsible for the decrease.
\end{abstract}

\section{Speculation}

Increasing cholinesterase activity during gestation may, in part, protect more mature fetal hearts from severe bradycardia. Likewise, "unprotected" AcH receptors may account for the pronounced bradycardia in less mature hearts. If unprotected AcH receptors are important to the bradycardic response, does this play any role in vivo? One possibility is that the fetal heart's capacity to synthesize AcH and acetylcholinesterase may not develop synchronously. Such nonparallel enzyme system development might occur as a normal developmental sequence, exposed abnormally by premature birth or conversely, may be induced by abnormalities during gestation ( e.g., chronic hypoxemia, intrauterine malnutrition). This general scheme is not unprecedented in that asynchronous development of interrelated enzymes systems deleterious to the prematurely born have already been well documented in the liver and lung. This hypothesis is currently being investigated.

The widespread use of heart rate monitoring in fetal and neonatal intensive care has revealed a rather high incidence of bradyarrhythmias in the newborn infant $(8,21,25,38,41,42)$. In fact, detection of bradycardia has become a major factor in determining the therapeutic threshold of certain fetal and neonatal afflictions $(13,17)$.

Clinical and laboratory investigations as well as simple clinical observations have suggested: 1) Bradycardia (usually sinus) occurs spontaneously and in response to a wide variety of stimuli such as increased CNS pressure, umbilical cord compression, hypoxemia, apnea, nasopharyngeal and laryngeal stimulation, defecation, star- tle and attention behavioral responses $(3,21,34,35,42) .2)$ The incidence and/or the severity of bradycardia may be increased in the premature infant $(18,26,27,34)$.

As a group, immature fetuses and neonates are certainly subject to more bradycardia-evoking stimuli. However, the occurrence and severity of this arrhythmia (after many stimuli) seems especially pronounced in the premature although conclusive clinical studies addressing this impression are lacking.

Despite ample documentation of the prevalance of bradyarrhythmias in the newborn, specific mechanisms are poorly defined and developmental changes in those mechanisms are equally unclear. Investigators have suggested that bradycardic episodes in neonates may be explained by immaturity of the heart's autonomic control $(4,20,21,27)$, inadequate CNS modulation of afferent stimuli affecting heart rate (42), imbalance of cholinergic and adrenergic neuroeffector competence (21), altered sensitivity to vagal afferent stimuli $(17,34)$, altered responsiveness of the myocardium to cholinergic stimuli (32), myocardial hypoxemia (10), and ontogenetic recapitulation of a "diving reflex" (21). The difficulty in isolating and defining any of these explanations of neonatal bradycardia in either a clinical or laboratory setting remains considerable.

Accordingly, I have attempted to define the pattern of intrinsic cardiac responsiveness to the neurotransmitter, $\mathrm{AcH}$, during development in the fetal mouse and to test some of the mechanisms responsible for this pattern.

Isolated, intact, beating fetal mouse hearts in organ culture provided an experimental system in which intrinsic cardiac responsiveness to neurotransmitters could be isolated and which fairly diverse fetal developmental stages were easily available.

\section{MATERIALS AND METHODS}

\section{PREPARATION OF ISOLATED HEARTS}

Pregnant Swiss albino white mice were obtained at the beginning of the third trimester from a local commercial laboratory animal supplier. Dating of pregnancy was accurate to within 24 hr of expected gestational length. Isolation of fetal hearts essentially followed Wildenthal's original description (44).

The pregnant mother was rapidly killed by cervical dislocation. Within about $1 \mathrm{~min}$, both gravid uterine horns were removed sterilely and placed in a $100 \mathrm{~mm}$ glass Petri dish containing Hank's balanced salt solution (HBSS) at approximately $4^{\circ} \mathrm{C}$.

The pregnant uterus was carefully incised longitudinally allowing gentle expulsion of fetuses without disruption of fetal membranes. Intact fetal mice were then removed by incision of membranes and umbilical cord. The fetal head was then cut off and the abdomen was transected, thus, leaving an isolated fetal thorax. After transfer to fresh, cold HBSS, the thorax was opened with a median sternotomy incision under a dissecting microscope. The heart, lungs, and thymus were then removed en block and transferred to fresh, cold HBSS in a $60 \mathrm{~mm}$ Falcon plastic Petri dish. 
Subsequently, under the dissecting scope, the attached lung, thymus, great vessels, connective tissue, and pericardium were dissected away leaving an isolated fetal heart. Incision, distortion, and grasping of the heart was carefully avoided. Isolated hearts were kept in cold HBSS before placement in culture.

Preparation of 10-12 isolated hearts requires approximately $l^{1 / 2}$ $\mathrm{hr}$ and each heart is kept at $4^{\circ} \mathrm{C}$ except for a $5-10 \mathrm{~min}$ period. Fetal hearts from gestations of 13-21 days are easily obtained by this procedure. Isolation of hearts below 13 days is possible, but more difficult and, therefore, more likely to cause damage to the heart.

Individual fetal heart weights were determined by measuring the combined weight of 2-3 isolated hearts after blotting off excess fluid. Weights ranged from $0.5-10.0 \mathrm{mg}$ during the gestational period studied. Heart weight and corresponding GA are presented in Table 1 . These figures are derived from an initial determination of heart weights in a group of animals (91 fetuses, 15 mothers) in which gestation was accurate to within 12-15 hr. Subsequent determination of the fetal heart's GA was on the basis of heart weight in that accurate timing of pregnancy would not allow supply of an adequate number of pregnant females. Heart weight and GA determinations are quite similar to those presented by Wildenthal (45).

\section{CULTURE CONDITIONS}

Fetal hearts were rinsed once in fresh HBSS after isolation to facilitate removal of any remaining intracavitary blood. Then, single, isolated hearts were pipetted onto a $0.5 \mathrm{~mm}$ mesh stainless steel grid situated at the media atmosphere interface within a 60 $\mathrm{mm}$ plastic Falcon organ culture dish containing exactly $0.5 \mathrm{cc}$ of nutritive media (HBSS with $4 \mathrm{mM}$ glucose or Medium 199) within the inner well. Each set of cultured littermate hearts was then placed in a gas tight, stainless steel cannister to which an atmosphere of $95 \% \mathrm{O}_{2} / 5 \% \mathrm{CO}_{2}$ was introduced to insure adequate oxygenation and maintenance of the media $\mathrm{pH}$ at 7.3-7.4. Cannisters and contained hearts were then incubated at $37 \pm 0.5^{\circ} \mathrm{C}$ for a total of 30-36 hr. Nutritive solutions were replaced with fresh media after $18-22 \mathrm{hr}$ in culture.

\section{NEUROTRANSMITTER ADDITION AND HEART RATE COUNTING}

After 30-36 hr in culture, organ culture dishes with contained hearts were transferred to a laser beam heart rate counting system for drug addition experiments. A detailed description of the rate counting system is available elsewhere (23). Briefly, the organ culture dish and contained fetal heart is placed in a $37^{\circ} \mathrm{C}$ miniature circulating water bath situated on a dissecting microscope substage. A laser beam is directed through the beating heart and deflections of the beam resulting from heart contractions are projected onto a small photocell through a pinhole aperture. Output from the photocell is received by a strip chart recorder and a permanent record of heart rate throughout the entire experiment is, thus, obtained.

Immediately after placement in the counting system, baseline heart rate and rhythm were determined. Differences in atrial and ventricular rates were assessed by appropriately positioning the laser beam. Two min of baseline heart rate were recorded and immediately followed by addition of $50 \mathrm{ul}$ of a drug containing

Table 1. Heart wt and corresponding GA's.

\begin{tabular}{cc}
\hline $\mathrm{Wt}_{\mathrm{t}}(\mathrm{mg})^{1}$ & GA (days) \\
\hline $0.5-1.0$ & $13-14$ \\
$1.1-2.0$ & $15-16$ \\
$2.1-4.0$ & $17-18$ \\
$4.1-6.0$ & $19-20$ \\
$6.1-10$ & $21-22$
\end{tabular}

\footnotetext{
'Range encompassing all measured weights in corresponding GA groups. $N=18$ each group.
}

solution. Six to seven rapid $50 \mathrm{ul}$ aspirations and additions after initial drug aliquot addition ensured complete and rapid mixing of drug with the heart's nutritive media. Drugs used in these experiments were acetylcholine chloride (Sigma), isoproterenol HcL (Sigma), physostigmine (Sigma), dl-propanolol HcL (Sigma), atropine sulfate (Sigma), and D-tubocurarine chloride (Sigma). All drug solutions were prepared immediately before use. An appropriate amount of drug was dissolved in $5 \mathrm{ml}$ of HBSS so that a $50 \mathrm{ul}$ aliquot of this stock solution would yield the desired drug concentration presented to the fetal heart after mixing with the heart's $0.5 \mathrm{cc}$ of nutritive media. Drug solutions were gassed with $95 \% \mathrm{O}_{2} / 5 \% \mathrm{CO}_{2}$ and warmed to $37^{\circ} \mathrm{C}$ before use. By manipulation of the stock solution drug concentration, serial additions to an individual heart resulted in exposure to progressively larger drug concentration and hence construction of dose response curves. More often, however, dose response curves were determined by single additions of a specific drug concentration to matched littermate hearts. Replacement of the entire quantity of the heart's nutritive media with an equal quantity of media containing the desired concentration of the drug was not used in that preliminary experiments revealed that the initial withdrawal of media caused bradycardia in many of the older GA hearts, thus, making interpretation of subsequent drug effect difficult.

Heart rate was counted continuously before, during, and after addition of the drug. Baseline heart rate at all GA's remains constant in the rate counting system for $15-20 \mathrm{~min}$ if no drug is added. Experimental sessions for each fetal heart lasted 5-10 min.

Only those hearts with identical atrial and ventricular rates $\geq 110$ and without arrhythmia during the baseline counting period were used.

After each counting session, fetal hearts used were inspected under a dissecting scope to insure that unsuspected physical damage (during isolation) or submersion in the media was not present. Results from any such hearts were discarded in that these conditions seem to compromise the heart's viability.

\section{STATISTICS}

Statistical analyses of the data were made with Student's $t$ test. Significance of drug induced changes at each specific drug concentration for hearts of different AG's was determined by the test for paired observations. Each heart's baseline rate served as its control. Significance of differences in dose response for each gestational age was determined using the test for grouped data.

\section{RESULTS}

Approximately 550 fetal hearts of various GA's were used in these experiments. Each data point represents the mean heart rate response of at least 10 different hearts at each GA (heart weight). Dose response curves were determined only from data on matched littermate hearts. Each heart served as its own control.

\section{BASELINE HEART RATES}

Baseline (or control) heart rate after $30-36 \mathrm{hr}$ in culture gradually increases with increasing GA. The highest initial heart rate observed was $253 \mathrm{bpm}$ in a term heart. As mentioned previously, hearts with baseline rates $<110$ were not used. Only approximately $5 \%$ of all hearts were discarded by this criterion. Another $5 \%$ could not be used due to arrhythmias or A-V dissociation at baseline. Exposure to the laser beam for up to $15 \mathrm{~min}$ was without effect on baseline heart rate at any $\mathrm{GA}$.

\section{AcH RESPONSE}

Addition of AcH to fetal hearts results in a fairly constant triphasic heart rate response. Initial onset of bradycardia occurs at $5-10$ sec after drug addition and is then followed by a $10-30 \mathrm{sec}$ period of exaggerated, transient slowing of the rate. A stable heart rate response to AcH begins at 45-60 sec and persists from 30-300 
seconds depending on $\mathrm{AcH}$ dose and GA. All determinations of heart rate response were made during this latter stable period.

Exposure of fetal hearts to $10^{-4}, 10^{-6}$, and $10^{-8} \mathrm{M} \mathrm{AcH}$ revealed a progressive decrease in the bradycardic response with increasing GA (Fig. I).

The difference in heart rate response with $\mathrm{GA}$ was most striking at a relatively high $10^{-4} \mathrm{M} \mathrm{AcH}$ with an $84 \%$ fall at 13-14 days

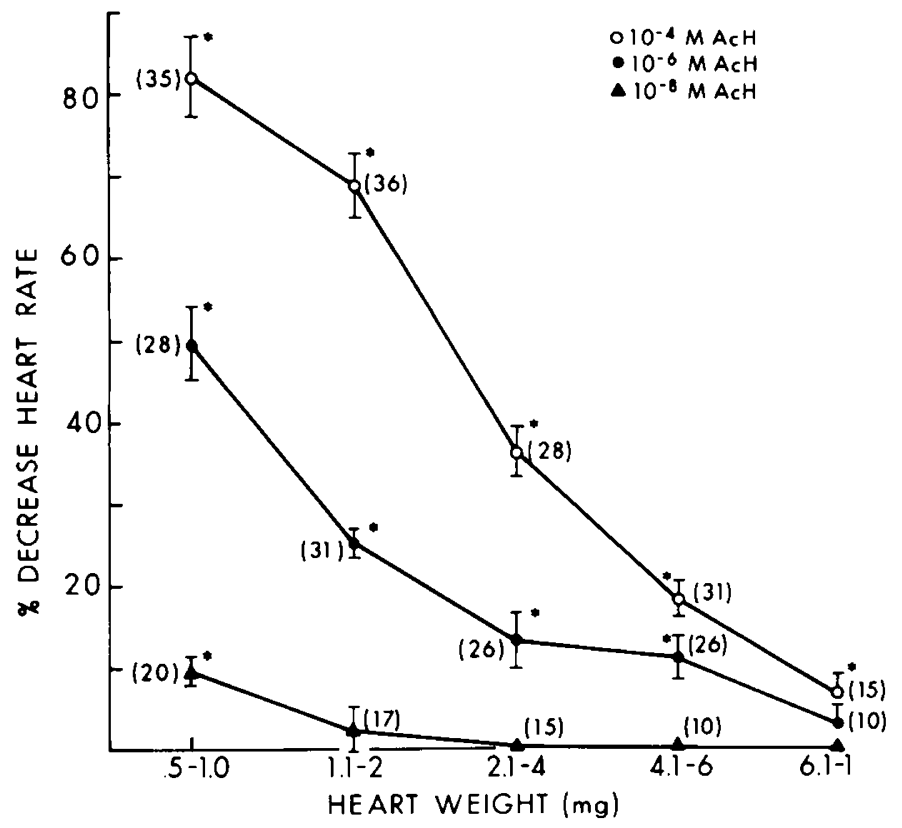

Fig. 1. Dose response curves to AcH during 13-21 days of gestation (heart wt 0.5-10 mg). Each data point represents mean $\pm \mathrm{SE}$ for percent fall in heart rate from baseline in a group of fetal hearts with weights in the range indicated on abscissa. Number of hearts per data point is in parentheses. Responses at different $\mathrm{AcH}$ doses were determined from matched littermate hearts. Asterisk indicates significant decret se from baseline rate $(P<0.001)$. and only a $7 \%$ decrease from baseline in the most mature hearts. The same trend of decreasing bradycardia with increasing GA is also evident with exposure to $10^{-6} \mathrm{M} \mathrm{AcH}$ although the magnitude of the response is diminished. Only the least mature hearts showed any response to $10^{-8} \mathrm{M} \mathrm{AcH}$.

Approximately one-third of the least mature hearts developed asystole after exposure to $10^{-4} \mathrm{M} \mathrm{AcH}$. None of the fetal hearts with weights greater than $2 \mathrm{mg}$ (GA $>16$ days) became asystolic at this dose. No evidence of atrial asystole without simultaneous ventricular asystole during the initial or stable response to $\mathrm{AcH}$ was noted. However, a few ventricular contractions frequently preceded atrial beats during recovery from $\mathrm{AcH}$ in youngest $\mathrm{GA}$ hearts. Atrioventricular block not resulting in dissociation could not be assessed.

Exposure of the hearts to $10^{-6} \mathrm{M}$ atropine before $\mathrm{AcH}$ addition completely abolished the heart rate response at all GA's and all molar concentrations of $\mathrm{AcH}$ (Fig. 2). Additionally, no increase in heart rate from baseline was observed in the most mature hearts after combined atropine and $\mathrm{AcH}$ exposure.

The possibility of AcH stimulated cardiac norepinephrine release was also assessed by exposure to $10^{-6} \mathrm{M}$ propanolol before $\mathrm{AcH}$ addition. $\beta$-blockade was without effect on $\mathrm{AcH}$ response at any $\mathrm{GA}$ after $36 \mathrm{hr}$ in culture.

A set of fetal hearts was also exposed to the nicotinic blocker, D-tubocurarine $\left(10^{-6} \mathrm{M}\right)$, before $\mathrm{AcH}$ addition. No significant effect on resultant $\mathrm{AcH}$ heart rate response was noted.

\section{PHYSOSTIGMINE RESPONSE}

It is possible that with increasing GA and, therefore, increasing parasympathetic innervation, augmented levels of acetylcholinesterase in more mature fetal mouse hearts may effectively blunt the bradycardic response to $\mathrm{AcH}$. To test this possibility, dose response curves to $10^{-4}$ and $10^{-6} \mathrm{M}$ AcH were repeated in the presence of the anticholinesterase, physostigmine, at $10^{-6} \mathrm{M}$ concentration (Fig. 3).

A significant increase in response to $\mathrm{AcH}$ was present in fetal hearts with weights from $2.1-10 \mathrm{mg}$ (or about 17-21 days GA). The most striking augmentation of the bradycardic response oc-

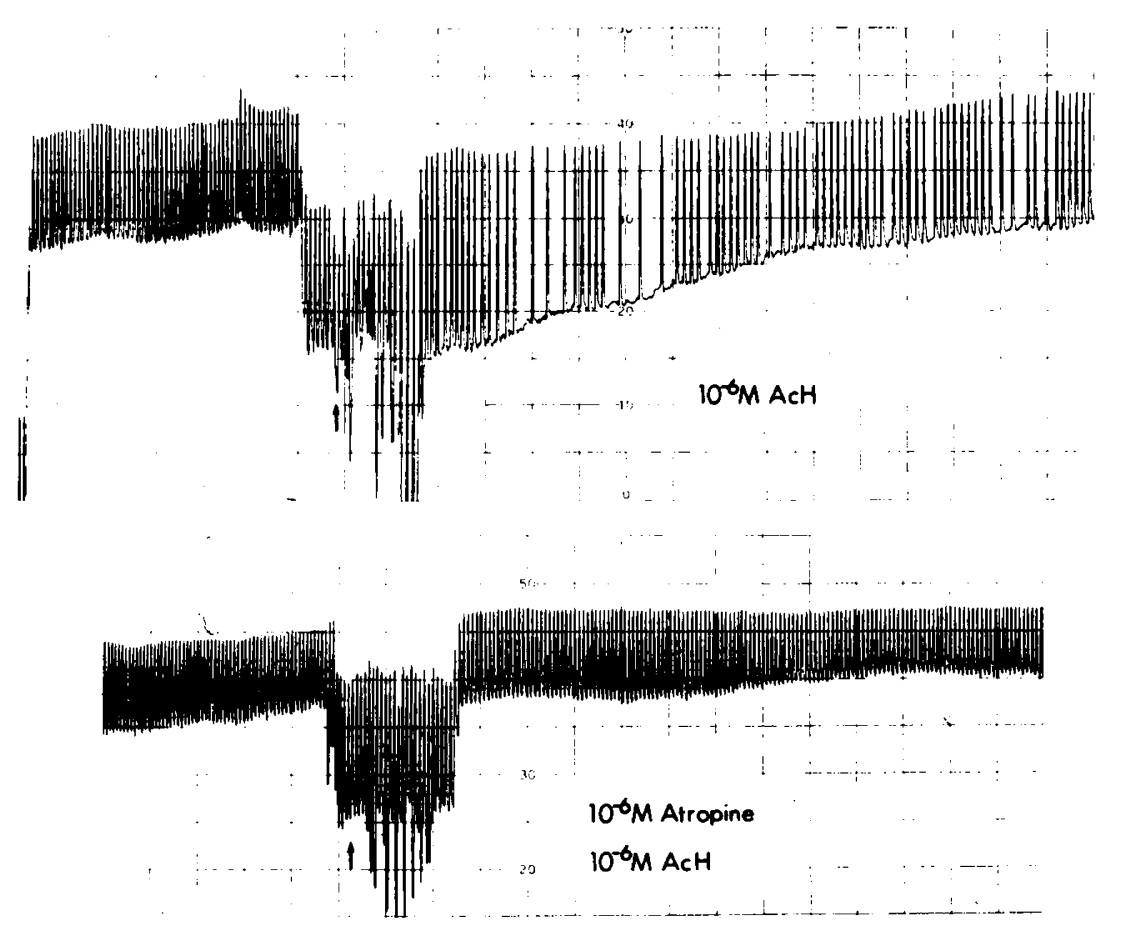

Fig. 2. Example of heart rate response to $10^{-4} \mathrm{M} \mathrm{AcH}$ with (lower tracing) and without (upper tracing) $10^{-6} \mathrm{M}$ atropine. Fetal heart weight $=3.6 \mathrm{mg}$ and estimated GA is 17-18 days. Arrow indicates AcH addition in each tracing. Each deflection indicates a single heart contraction. The height of the deflection has no particular meaning. 


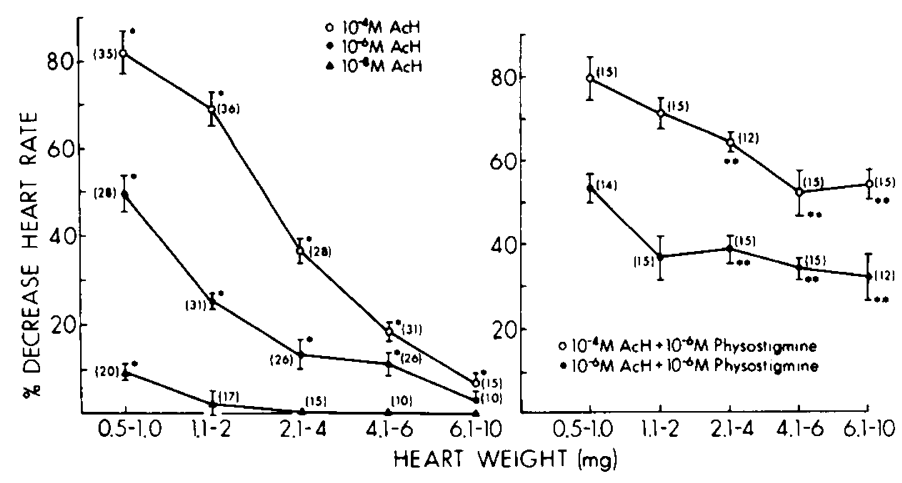

Fig. 3. Dose response curves to $\mathrm{AcH}$ with (on right) and without (on left) $10^{-6} \mathrm{M}$ physostigmine. Data points are mean $\pm \mathrm{SE}$ percent of decrease in heart rate from baseline. Responses at different $\mathrm{AcH}$ concentrations with and without physostigmine are from matched littermates. Number of hearts per data point is in parentheses. * indicates significant decrease from baseline rate $(P<0.001){ }^{* *}$ indicates significant difference in heart rate response compared to response with $\mathrm{AcH}$ alone $(P<0.001)$.

curred in the most mature hearts. In this group $10^{-4} \mathrm{M} \mathrm{AcH}$ caused only a $7 \%$ heart rate fall without anticholinesterase, but a $54 \%$ decrease with physostigmine. Corresponding values for $10^{-6}$ $\mathrm{M} \mathrm{AcH}$ are $3 \%$ before physostigmine and $32 \%$ after.

No significant difference in response to AcH was seen in less mature hearts after physostigmine addition. A small $(<10 \%)$ transient slowing of heart rate was noted in fetal hearts of $2 \mathrm{mg}$ or less after exposure to $10^{-6} \mathrm{M}$ physostigmine alone. Return to baseline rate always occurred within $60 \mathrm{sec}$ and $\mathrm{AcH}$ addition was delayed until that time. No isolated effects of physostigmine were observed in larger (older) hearts.

\section{ISOPROTERENOL RESPONSE}

Dose response curves to isoproterenol were also established to determine the change in responsiveness to an autonomic effector expected to elicit a chronotropic response opposite to that of $\mathrm{AcH}$. More specifically, limitations in heart rate response due to differences in receptor site accessibility between younger (smaller) and older (larger) hearts could be tested by comparing isoproterenol responses to $\mathrm{AcH}$ responses (Fig. 4). Exposure to $10^{-4} \mathrm{M}$ isoproterenol resulted in a minimal increase $(14 \%)$ in least mature hearts. Tachycardia in response to this dose progressively increased with increasing GA culminating in a $123 \%$ increase in the most mature hearts. Heart rate increases after $10^{-6} \mathrm{M}$ isoproterenol amounted to $6 \%$ at $13-14$ days $(0.5-1.0 \mathrm{mg})$ and $75 \%$ at $20-21$ days $(6.1-10$ $\mathrm{mg}$ ). Onset of heart rate response at all GA's was between 5-15 sec with maximum stable response occurring at 50-60 sec after drug addition. Preaddition of $10^{-6}$ propanolol in three hearts of each GA completely abolished response to isoproterenol.

\section{SHAM DRUG ADDITION}

Sham "drug" addition of $50 \mathrm{ul}$ pregassed, $37^{\circ} \mathrm{C}$ HBSS to approximately 10 hearts of each GA resulted in no significant heart rate changes from baseline over a 5-min testing period.

\section{DISCUSSION}

Exposure of isolated, fetal mouse hearts in organ culture to varying concentrations of $\mathrm{AcH}$ results in a progressively more marked heart rate slowing with decreasing GA. The marked bradycardia induced in the youngest hearts (14-15 days GA) is especially remarkable in that cardiac parasympathetic innervation is presumably either not present or is just beginning at this age $(30,45)$.

If conventional pharmacologic mechanisms apply, atropine sensitive responsiveness to acetylcholine (even before innervation)

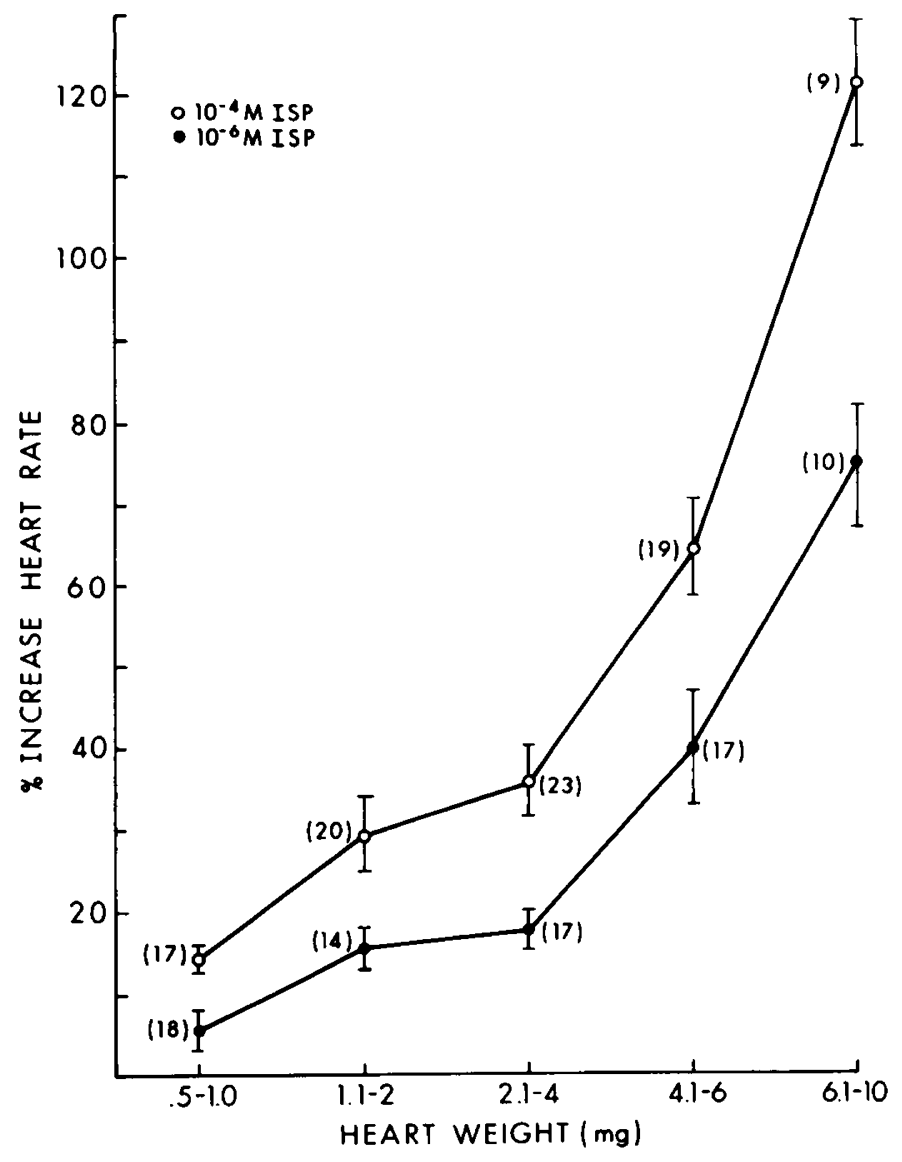

Fig. 4. Dose response curves to isoproterenol from 13-22 days GA (0.5-10 mg heart wt). Data points are mean $\pm S E$ of percent of increase in heart rate from baseline. Responses at different isoproterenol concentrations are from matched littermate hearts. Number of hearts per data point is in parentheses.

must imply functional muscurinic acetylcholine receptors and an operative, chronotropic receptor-response coupling. Investigations of chronotropic responsiveness in hearts from fetal rats, $(12,36)$ chicks, $(6,14)$ mice $(45)$ and humans $(42)$ have demonstrated $\mathrm{AcH}$ induced heart rate slowing before morphologic parasympathetic innervation. Furthermore, Roeske and Yamanura (37) and Sastre et al. (39) have detected AcH receptors before innervation by labeled competitive antagonist binding. Also, myocardial membrane potassium and sodium conductance changes typical of well documented $\mathrm{AcH}$ effects in adult hearts (40) have been noted in chick (33) and rat (31) hearts at or before innervation.

It thus appears well established that myocardial responsiveness to $\mathrm{AcH}$ is present before or at the very onset of recognizable morphologic parasympathetic innervation. However, the apparently exaggerated responsiveness to $\mathrm{AcH}$ noted in the least mature hearts in the present investigation is more difficult to explain. Indeed, other investigators using the mouse $(37,45)$ as well as other species $(12,32,36)$ suggest that $\mathrm{AcH}$ response becomes progressively greater with increasing GA and increasing parasympathetic innervation. Variability in species, developmental age, specific methodology of preparation and preservation of fetal hearts, AcH dose, and experimental design make comparisons of $\mathrm{AcH}$ dose response relationships difficult.

Previous studies demonstrating greater responsiveness with increasing parasympathetic innervation have generally related such a pattern to the gradual accruance of $\mathrm{AcH}$ receptor sites $(37,39)$ and/or increasing receptor-response sensitivity $(31,33,45)$. Certainly receptor site density, sensitivity and affinity for neurotransmitters along with several other components of neuroeffector physiology may undergo developmental change and, thus, explain 
different age-related dose responses. All such elements of the neurotransmitter/effector/response relationship interact to produce a specific response. Moreover, the rate and course of maturation of each component may differ (32). For instance, it is not necessarily true that receptor sensitivity or even ultimate effector response increase in parallel with receptor site density. Experiments by Friedman (7) and Brus and Jacobowitz (1) reveal that receptor sensitivity to $\mathrm{AcH}$ may remain unchanged or diminish during the elaboration of the heart's parasympathetic innervation. The pattern of $\mathrm{AcH}$ response noted in the present investigation may also result from an initial high receptor-response sensitivity and, thereafter, progressive diminution of sensitivity with increasing GA and innervation. A decrease in receptor-response sensitivity as intrinsic cardiac neural capacity for production and release of $\mathrm{AcH}$ increases might serve to modulate the parasympathetic stimulus/response relationship during development. There is some support for this general scheme in that subsensitivity to catecholamine apparently develops during sympathetic innervation of the dog heart (2). Changes in receptor sensitivity as well as local metabolism of neurotransmitter were thought to explain the developmental pattern in that study. Furthermore, increases in receptor site density may or may not compensate for loss in sensitivity. It should be noted, however, that the developmental pattern of receptor-response sensitivity has not been precisely defined for the fetal mouse heart in this or other studies.

Intracardiac neuronal release of endogenous $\mathrm{AcH}$ (stimulated by exogenous application of $\mathrm{AcH}$ ) has also been suggested as a possible explanation for augmented heart rate slowing in gestationally older embryonic chick hearts with more extensive parasympathetic innervation (22). This mechanism might explain the similar $\mathrm{AcH}$ responses noted in previous studies using fetal mouse hearts $(37,45)$. However, pharmacologic release of intrinsic $\mathrm{AcH}$ in the chick or fetal mouse heart has not been conclusively demonstrated. Documentation of such intrinsic neurotransmitter effects requires measurement of endogenous AcH content and possibly choline acetyltransferase activity along with appropriate field stimulation experiments to confirm neuronal mediation of $\mathrm{AcH}$ release.

In addition to the rather extreme chronotropic response to $\mathrm{AcH}$ in the least mature hearts, a progressive decrease in response with increasing GA was noted in this investigation. These response changes occur during a period of presumptive increasing morphologic and functional parasympathetic innervation $(30,45)$. Histologic and biochemical studies in human (29), dog (5), and cat (15) hearts suggest that acetylcholinesterase is closely linked to the morphologic features of parasympathetic innervation (e.g., neuroeffector junctions, cardiac ganglia). Furthermore, the amount and distribution of acetylcholinesterase apparently increase in concert with developing cardiac innervation $(19,24)$. There is evidence that cholinesterase activity is detectable before innervation (11) and speculation that it may even induce and direct subsequent parasympathetic nerve ingrowth (16). By inference from other studies $(28,29)$, it is probable that cardiac ganglia and parasympathetic postganglionic cardiac nerves remain intact in organ cultured fetal mouse hearts. If acetylcholinesterase activity is retained at $\mathrm{AcH}$ sensitive sites (SA node, AV node, atrial myocardium), increasing cholinesterase activity during cardiac maturation may serve to progressively limit chronotropic $\mathrm{AcH}$ response.

Accordingly, inhibition of cholinesterase activity with physostigmine resulted in a significant augmentation of the bradycardic response to $\mathrm{AcH}$ in older $\mathrm{GA}$ hearts. The shorter duration of induced heart rate slowing in more mature hearts also suggests indirectly that developmental changes in cholinesterase activity play a role in shaping AcH response. The lack of anticholinesterase augmentation of bradycardia in the youngest hearts probably related to a rather small amount of acetylcholinesterase confronted with a large AcH stimulus. A similar developmental pattern of cholinesterase modulation of $\mathrm{AcH}$ response has been demonstrated in fetal rabbit hearts (1).

It is possible that the implied cholinesterase activity in this study is not due to neural acetylcholinesterase specifically. Pseudocho- linesterase or fetal cholinesterases not linked to parasympathetic innervation could also inhibit $\mathrm{AcH}$ response although the rate of degradation of $\mathrm{AcH}$ by such nonspecific cholinesterases is much slower than by acetylcholinesterase. This possibility is difficult to exclude. However, one obvious source of noncardiac cholinesterase activity, retained fetal blood, was eliminated by careful evacuation of blood during isolation of fetal hearts and by exchange of nutrient media before testing.

Although inhibition of cholinesterase activity seems to explain, in part, the developmental changes in $\mathrm{AcH}$ dose response, some other possible contributing factors were explored.

It is possible that the observed AcH effects on heart rate were due to a nonspecific depression of pacemaker activity or even stimulation of nicotinic rather than muscurinic receptor sites. However, AcH response was abolished by the muscurinic blocker, atropine, at all GA's and all AcH concentrations. Additionally, nicotinic blockade with D-tubocurarine was without effect on dose response curves. The lack of change in AcH response with nicotinic blockade also undermines the possible contribution of $\mathrm{AcH}$ stimulated, cardiac ganglia mediated norepinephrine release as a modulating influence on the development of bradycardia in older fetal mouse hearts. AcH also stimulates release of norepinephrine from intracardiac adrenergic nerves (2). Heart rate changes from this mechanism would be $\beta$-blockade sensitive and inclusion of propanolol was without significant effect on AcH response. Furthermore, at $36 \mathrm{hr}$ in culture, intracardiac norepinephrine stores are significantly depleted (45).

One other major possibility for the diminished response to $\mathrm{AcH}$ in more mature fetal mouse hearts might be simply on the basis of their greater size. The heart's viability as well as access to neuroeffector substances is directly dependent on diffusion rather than perfusion in this organ culture system. It is, therefore, possible that diffusion limitations imposed by a larger cultured heart might affect response to $\mathrm{AcH}$ either on the basis of limited accessibility to receptor sites or because of a more marginal general condition of the heart.

To test the first possibility, the developmental pattern of heart rate response to isoproterenol was determined. If receptor site inaccessibility limits response to $\mathrm{AcH}$, one might expect a similarly diminished response to isoproterenol. Instead, a progressive increase in heart rate elevation occurs during this 13-21 day gestational period. A very similar pattern was demonstrated using isoproterenol and norepinephrine by Wildenthal (45).

Also, the very similar time course of response onset and maximum stable response in both the youngest and oldest fetal hearts to AcH and isoproterenol militates against any significant diffusion limitation in larger hearts.

Finally, use of response data only from hearts demonstrating spontaneous, nearly normal baseline beating rates, without A-V dissociation, other spontaneous arrhythmias, or physical evidence of damage during isolation argues against compromise of the heart's viability as an explanation for neurotransmitter response differences. Previous morphologic studies of fetal mouse hearts suggest that the cultured heart slowly deteriorates over time, gradually losing a small amount of weight each day (44). Despite this, regular, spontaneous, rapid beating is maintained for up to 14 days depending on specific culture conditions (44). Sponteneous, baseline heart rate does not change significantly until 5-7 days in hearts of any GA using culture conditions outlined in this report. Selection of a $30-36 \mathrm{hr}$ culture period before testing was somewhat arbitrary, but seems well within the 5-7 day viability limitations. Also, preliminary experiments with hearts cultured for only 3-4 hr revealed a high frequency of spontaneous arrhythmias and periods of quiescence in older gestational aged hearts which disappeared in most by $12-24 \mathrm{hr}$. Drug response in such unstable hearts would have been difficult to interpret.

\section{COMMENT}

This investigation outlines the developmental pattern of the fetal mouse heart's intrinsic responsiveness to the neurotransmitter $\mathrm{AcH}$. Increasing cholinesterase activity during gestation seems to 
account for this pattern, in part, and may protect more mature fetal hearts from severe bradycardia.

Although results from in vitro experiments with fetal mouse hearts can not be freely extrapolated to developing human fetuses, the propensity for bradyarrhythmias in immature human infants coupled with the striking bradycardia demonstrated in immature fetal mouse hearts is intriguing. The importance of other determinants of cholinergic responsiveness such as central nervous system maturation or the balance of cholinergic and adrenergic competence during gestation should certainly not be discounted. Rather, this and other investigations simply suggest that when central nervous system participation in cholinergic responsiveness is eliminated, intrinsic cardiac responsiveness to pharmacologic cholinergic stimulation markedly changes during development.

\section{REFERENCES AND NOTES}

I. Brus, R., and Jacobwitz, D.: The influence of norepinephrine, tyramine, and acetylcholine upon isolated perfused hearts of immature and adult rabbits. Arch. Int. Pharmac. Ther., 200: 266 (1972).

2. Burn, J. H., and Rand, M. J.: Acetylcholine in adrenergic transmission. Annu. Rev. Pharmacol., 5: 163 (1965).

3. Cardero, L., and Hon, E. H.: Neonatal bradycardia following nasopharyngeal stimulation. J. Pediatr., 78: 441 (1971).

4. Church, S. A., Morgan, B. C., Oliver, T. K., and Guntheroth, W. G.: Cardiac arrhythmias in premature infants: an indication of autonomic immaturity? J Pediatr., 71: 542 (1967)

5. Cooper, T.: Terminal innervation of the heart. In: W. Randall: Nervous Contro of the Heart. p. I30-153 (The Williams \& Wilkins Company, Baltimore, 1965).

6. Cullis, W. C., and Lucas, C. L.: Action of acetylcholine on the aneural chick heart. J. Physiol. (London), 86: 53P (1936).

7. Friedman, W. F.: The intrinsic physiologic properties of the developing heart Prog. Cardiovasc. Dis., 15: 87 (1972).

8. Gabriel, M., and Albani, M.: Cardiac slowing and respiratory arrest in preterm infants. Eur. J. Pediatr., 122: 257 (1976)

9. Geis, W. P., Tatooles, C. J., Priola. D. V., and Friedman, W. F.: Factors influencing neurohumoral control of the heart in the newborn dog. Amer. J. Physiol., 228: 1685 (1975).

10. Girling, D. J.: Changes in heart rate, blood pressure, and pulse pressure during apneic attacks in newborn babies. Arch. Dis. Childhood, 47: 405 (1972).

11. Gyévai, A.: Comparative histochemical investigations concerning prenatal and postnatal cholinesterase activity in the heart of chickens and rats. Acta Biol. Acad. Sci. Hung., 20: 253 (1969).

12. Hall, E. K.: Acetylcholine and epinephrine effects on the embryonic rat heart. J. Cell. Comp. Physiol., 49: 187 (1957).

13. Hon, E. H., Zannini, D., Quilligan, E. J.: The neonatal value of fetal monitoring. Am. J. Obstet. Gynecol., 122: 508 (1975).

14. Hsu, F. -Y.: The effect of adrenalin and acetylcholine on the heart rate of the chick embryo. Chin. J. Physiol., 7: 243 (1933).

15. Jacobowitz, K., Cooper, T., and Barner, H. B.: Histochemical and chemical studies of the localization of adrenergic and cholinergic nerves in the normal and denervated cat heart. Circ. Res., 20: 289 (1967).

16. Karczmar, A. G., Srinivasin, R., and Bernsohn, J.: Cholinergic function in the developing fetus. In: L. Borens: Fetal Pharmacology. p. 127-176 (Raven Press, New York, 1973).

17. Kattwinkel, J., Fanaroff, A. A., Klaus, M.: Bradycardia in preterm infants: Indications and hazards of atropine therapy. Pediatrics, 58: 494 (1976)

18. Kero, P.: Heart rate patterns in infants with respiratory distress syndrome. Acta Paediat. Scand. Suppl., 250: 1 (1974).

19. Lelomer, J., and Shickman, F. E.: Acetylcholinesterase and responses to acetylcholine in the embryonic chicken heart. J. Pharm. Pharmocol., 28: 196 (1976).

20. Lipton, E. L., Steinschneider, A., and Richmond, J. B.: Autonomic function in the neonate. VIII. Cardio-pulmonary observations. Pediatrics, 33: 212 (1964).

Copyright (C) 1979 International Pediatric Research Foundation, Inc. $0031-3998 / 79 / 1309-1052 \$ 02.00 / 0$
21. Lipton, E. L., Steinschneider, A., and Richmond. J. B.: The autonomic nervous system in early life. N. Engl. J. Med., 273: 147 (1963).

22. Loffelholz, K., and Pappano, A. J.: Increased sensitivity of sinoatrial pacemaker to acetylcholine and to catecholamine at the onset of acetonomic neuroeffector transmission in chick embryo heart. J. Pharmacol. Exp. Ther.. 191: 479 (1974).

23. Maurer, M., Yuhas, D., and Miller, J.: Laser beam method for measuring heart rate in organ cultured fetal hearts. J. Molec. Cell. Cardiol.. 11: 319 (1979)

24. McCarty, L. P., Lee, W. C.. and Shickman, F. E.: Measurement of the ionotropic effects of drugs on the innervated and noninnervated embryonic chick heart. J. Pharmacol. Exp. Ther., 129: 315 (1960).

25. Michaëlson, M.: Electrocardiographic studies in the healthy newborn. Acta Paediatrica 48 (Suppl. 117): 108 (1951)

26. Morgan, B. C., Bloom, R. S., and Guntheroth, W. G.: Cardiac arrhythmias in premature infants. Pediatrics, 35: 658 (1965).

27. Morgan, B. C.. and Guntheroth. W. G.: Cardiac arrhythmias in normal newborn infants. J. Pediatr.. 67: 1199 (1965)

28. Napolitano, L., Cooper. T.. William. V. L.. and Hanlon, C. R.: Fine structure of the heart after transplantation, with special reference to the neural elements. Circulation (Suppl. 1) 29: 81 (1964).

29. Napolitano, L., William. V. L., Hanlon. C. R. and Cooper. T.: Intrinsic innervation of the heart. Amer. J. Physiol.. 208: 455 (1965).

30. Otis, E. M., Brent, R.: Equivalent ages in mouse and human embryos. Anat Rec., /20: 33 (1954).

31. Pager, J., Bernard, C. and Cargouil, Y.-M.: Evolution au cours de la croissance foetale, des effets de l'acetylcholine au niveau de l'oreillette du rat. C. R. Soc. Biol. (Poitiers), 159: 2470 (1965)

32. Pappano, A. J.: Ontogenetic development of autonomic neuroeffector transmission and transmitter reactivity in embryonic and fetal hearts. Pharmacol. Rev.. 29: 3 (1977).

33. Pappano, A. J.: Sodium-dependent depolarization of non-innervated embryonic chick heart by acetylcholine. J. Pharmacol. Exp. Therap., 180: 340 (1972).

34. Phillips, S. J., Agate, F. J., Jr., Silverman. W. A., and Steiner, P.: Autonomic cardiac reactivity in premature infants. Biologia Neonatorum 6: 225 (1964).

35. Rigatto, H., and Brady, J. P.: Periodic breathing and apnea in preterm infants.: II. Hypoxia as a primary event. Pediatrics, 50: 219 (1972).

36. Robkin. M. A., Shepard. T. H., and Dyer. D. C.: Autonomic receptors of the early rat embryo heart: Growth and development. Proc. Soc. Exp. Biol. Med. 15I: 799 (1976)

37. Roeske, W. R., and Yamanura, H. I.: Maturation of myocardial muscurinic cholinergic receptors in the fetal mouse heart. Circulation Suppl III: Abstract 911 (1977).

38. Rudolph, A. J. Vallbona, ., and Desmond, M. M. Cardiodynamic studies in the newborn. III. Heart rate patterns in infants with idiopathic respiratory distress syndrome. Pediatrics, 36: 551 (1965).

39. Sastre, A., Gray, D. B. and Lane, M. A.: Muscurinic cholinergic binding sites in the developing avian heart. Develop. Biol., 55: 201 (1977).

40. Trautwein, W.: Generation and conduction of impulses in the hearts as affected by drugs. Pharmoacol. Rev., 15: 277 (1963).

41. Välimäki. I., and Tarlo, P. A.: Heart rate patterns and apnea in newborn infants Amer. J. Obstet. Gynecol., 110: 343 (1974).

42. Vallbona, C., Desmond, M. M.. Rudolph. A. J.. Pap, L. F., Hill. R. M. Franklin R. R.. and Rush, J. B.: Cardiodynamic studies in the newborn. II. Regulation of the heart rate. Biol. Neonate. 5: 159 (1963).

43. Walker, D.: Functional development of the autonomic innervation of the human fetal heart. Biol. Neonate, 25: 31 (1975).

44. Wildenthal. K.: Long-term maintenance of spontaneously beating mouse hearts in organ culture. J. Appl. Physiol.. 30: 153 (1971)

45. Wildenthal, K.: Maturation of responsiveness to cardioactive drugs. J. Clin Invest., 52: 2250 (1973).

46. The author thanks Dr. Burton Sobel, Washington University School of Medicine/ Cardiology, for the provision of laboratory space, financial support, and guidance for this investigation. I am indebted also to Dr. Philip Dodge. St. Louis Children's Hospital, for continued interest and support of my work. The author also gratefully acknowledges the instruction in the technique of isolating fetal mouse hearts provided by Dr. Jo Ann Ingwal. San Diego. California.47. Received for publication May 26, 1978.

48. Accepted for publication September 7, 1978. 